\title{
Lamin A/C mutations in dilated cardiomyopathy
}

\author{
Frédérique Tesson ${ }^{1}$, Michał Saj ${ }^{2}$, Musfira Mohamed Uvaize ${ }^{3}$, \\ Hannah Nicolas ${ }^{3}$, Rafał Płoski ${ }^{4}$, Zofia Bilińska ${ }^{5}$ \\ ${ }^{1}$ Faculty of Health Sciences, University of Ottawa, Ottawa, ON, Canada \\ ${ }^{2}$ Laboratory of Molecular Biology, Institute of Cardiology, Warsaw, Poland \\ ${ }^{3}$ Department of Biochemistry, Microbiology and Immunology, University of Ottawa, Ottawa, Canada \\ ${ }^{4}$ Department of Medical Genetics, Centre of Biostructure, Medical University of Warsaw, Warsaw, Poland \\ ${ }^{5}$ Unit for Screening Studies in Inherited Cardiovascular Diseases, Institute of Cardiology, Warsaw, Poland
}

\begin{abstract}
Dilated cardiomyopathy (DCM) is one of the leading causes of heart failure and heart transplant. Mutations in 60 genes have been associated with DCM. Approximately 6\% of all DCM cases are caused by mutations in the lamin A/C gene (LMNA). LMNA codes for type-V intermediate filaments that support the structure of the nuclear membrane and are involved in chromatin structure and gene expression. Most LMNA mutations result in striated muscle diseases while the rest affects the adipose tissue, peripheral nervous system, multiple tissues or lead to progeroid syndromes/overlapping syndromes. Patients with LMNA mutations exhibit a variety of cellular and physiological phenotypes. This paper explores the current phenotypes observed in LMNA-caused DCM, the results and implications of the cellular and animal models of DCM and the prevailing theories on the pathogenesis of laminopathies. (Cardiol J 2014; 21, 4: 331-342)
\end{abstract}

Key words: genetics, dilated cardiomyopathy, LMNA

\section{Introduction}

Dilated cardiomyopathy (DCM) is a disease of the heart muscle characterized by the dilatation of the left or both ventricles and reduced systolic function in the absence of abnormal loading conditions (hypertension, valve disease) or coronary artery disease (CAD) sufficient to cause global systolic impairment [1]. DCM is a significant health concern. It is the third most frequent cause of heart failure in the United States after CAD and hypertension [2]. Furthermore, DCM is also a primary indication for heart transplantation [2] and is marked by considerable morbidity as well as mortality. It is believed that $20 \%$ to $50 \%$ of idiopathic dilated cardiomyopathy (IDC) cases have familial causation [3, 4]. So far, more than 60 genes including the lamin $\mathrm{A} / \mathrm{C}$ gene (LMNA) have been associated with DCM. The pattern of the disease inheritance is mostly autosomal dominant [4]. Despite recent technological progress that makes gene screening both less time-consuming and cost-efficient, genetic screening currently reveals that only 30-35\% of familial DCM follow the Mendelian model of disease inheritance [5], while the remaining have a more complex multi-variant origin, which also encompasses the non-rare variants. In majority of the cases, incomplete age-related penetrance is observed [6-8]. It was reported that $7 \%$ of LMNA mutation carriers exhibit cardiac-related phenotypes if under 20 years of age, $66 \%$ when carriers are between 20 and 39 years, $86 \%$ when carriers are between 40 and 59 years, and $100 \%$ when carriers are over 60 years of age [6]. Another complicating factor that clouds the genetic diagnosis is the variability of expression within one phenotype. While some mutation carriers may develop all the symptoms of the disease, other family

Address for correspondence: Dr Frédérique Tesson, Faculty of Health Sciences, University of Ottawa, 451 Smyth Road, Ottawa, ON, Canada K1H 8M5, Canada, e-mail: ftesson@uOttawa.ca

Received: 28.07.2013 Accepted: 10.04.2014 


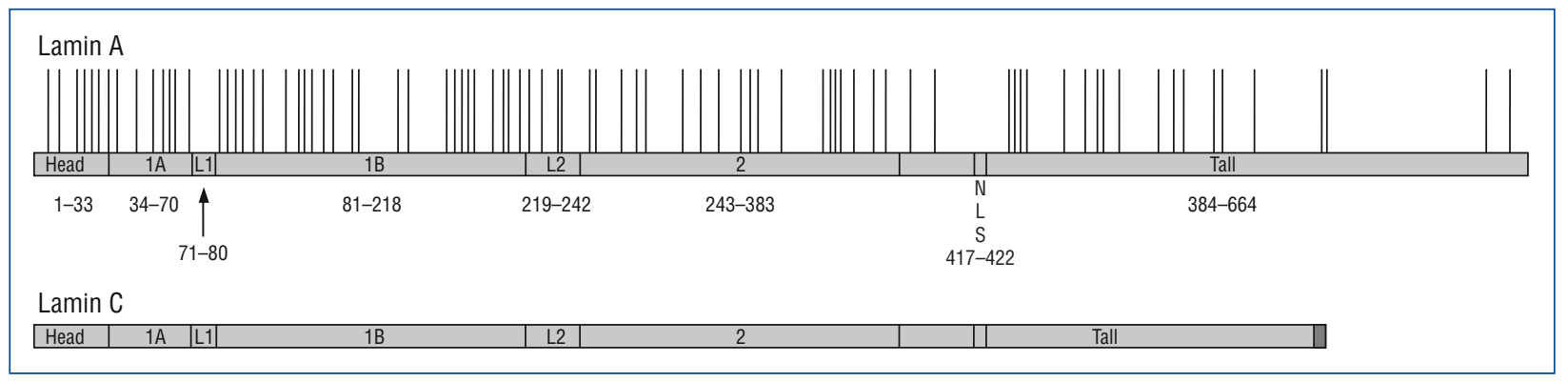

Figure 1. Protein map of lamin A and lamin $C$ with currently known mutations of both transcripts plotted onto lamin $A$ protein; NLS - nuclear localization signal. Dark shaded area at the C-terminal of lamin C represents lamin C-unique sequence.

members carrying the mutation exhibit only some aspects of it and may remain with a subclinical form of the disease. The onset of DCM can vary greatly as can the severity and the rate of progression of the disease. The variability may also pertain to the range of phenotypes such as in the case of 960delT LMNA mutation, which presented with 3 differing phenotypes within 1 family: pure DCM, DCM with Emery-Dreifuss muscular dystrophy (EDMD)-like symptoms and DCM with limb girdle muscular dystrophy (LGMD)-like symptoms [9].

Mutations in LMNA were first identified in a family with EDMD in 1999 [10]. In the same year, the association between LMNA mutations and DCM was reported [11]. Since then, an ever-growing number of mutations in LMNA have been identified defining a group of diseases called laminopathies. Laminopathies can be divided according to the observed phenotype. Most LMNA mutations have been associated with striated muscle diseases $(79.1 \%)$, followed by adipose tissue $(8.6 \%)$ and peripheral nervous tissue disorders $(0.3 \%) .9 .3 \%$ of LMNA mutations lead to progeroid syndromes while $10.9 \%$ cause overlapping syndromes with multiple tissue involvement [12].

Table 1 encompasses the most current list of LMNA mutations that lead to DCM, either isolated, with sole cardiac features or as a part of diagnosis of other, more complex conditions commonly affecting skeletal muscle such as EDMD or LGMD, but also encompassing other tissues which for instance leads to Charcot-Marie-Tooth disease, familial partial lipodystrophy, general lipodystrophy, hypogonadism, Hutchinson-Gilford progeria syndrome or diabetes mellitus. Table 1 also summarizes the span of phenotypic traits reported to be associated with a given mutation. It was created by combining information from four main LMNA mutation databases: the Human Intermediate Filament Database
[13], the Leiden Muscular Dystrophy website (www.dmd.nl), the $\mathrm{HGMD}^{\circledast}$ Professional 2012.4, the Universal Mutation Database (www.umd.be/ LMNA/) and from the literature. We were able to find 165 LMNA mutations leading to DCM (Fig. 1).

LMNA gene encodes the A-type lamins which are involved in maintaining the structural integrity of the nucleus, chromatin organization and gene expression [14]. LMNA is composed of 12 exons and encodes lamin $\mathrm{A}$ and lamin $\mathrm{C}$ by alternative splicing in exon 10 [15]. Both lamin isoforms are identical for the first 566 amino acids after which lamin $\mathrm{C}$ contains a unique sequence of 5 basic amino acids while amino acids from 567 to 664 are unique to lamin A [15]. In addition, prelamin A contains a CaaX motif at the $\mathrm{COOH}$-terminal, which undergoes posttranslational modifications [15]. Lamins are divided into 3 domains: a short globular head, an $\alpha$-helical rod and a globular tail. The rod domain comprises several coiled-coil domains separated by linker regions which are evolutionarily highly conserved (Fig. 1) [16].

Most lamin mutations leading to DCM are found in the head and rod domains covering more than half of lamin A and two-thirds of lamin C. DCM mutations are rarely found in the tail domain which contains many phosphorylation sites as opposed to mutations linked to EDMD, familial partial lipodystrophy and Hutchinson-Gilford progeria syndrome ([13]; Human Genome Mutation Database). However, hot spot(s) for DCM or other diseases affecting the striated muscle cannot be identified. Conversely, in adipose tissue defects, approximately $80 \%$ of cases carry a substitution of the p.Arg482 residue while $85 \%$ of mandibuloacral dysplasia cases are caused by a homozygous mutation at the p.527 residue and $77 \%$ of HGPS patients carry the c. $1827 \mathrm{C}>\mathrm{T}$ substitution within exon 11 [16]. 


\section{Studies of cellular phenotypes associated with LMNA mutations}

DCM patients with LMNA mutations display highly variable cardiomyocyte phenotype. A DCM patient encompassing exons 3-12 deletion showed diminished lamin $\mathrm{A}$ and $\mathrm{C}$ staining in the endomyocardial biopsy with discontinuous nuclear envelopes and invasion of mitochondria into the nuclear space [17]. Another DCM patient carrying a LMNA mutation displayed dramatic morphological alterations in approximately $30 \%$ of the cardiomyocyte's nuclei including a complete loss of the nuclear envelope [18]. However, other mutation carriers did not present with such dramatic abnormalities $[17,18]$. Nevertheless, cardiomyocytes from DCM patients with LMNA mutations usually display reduced lamin $\mathrm{A}$ and $\mathrm{C}$ in the nuclei with nuclear membrane damage such as focal disruptions, blebs and nuclear pore clustering $[19,20]$.

Skin fibroblasts isolated from patients with cardiac-or-skeletal-specific laminopathies most often had abnormal nuclear shape including blebs and herniation [21]. Lamin A and C distribution were affected in these cells and were either present in a honeycomb pattern [21] or distributed unevenly along the inner nuclear lamina [22]. Some fibroblasts had lamin $\mathrm{A}$ and $\mathrm{C}$ aggregates close to the lamina which did not interact with emerin, DNA or RNA [23]. Patient tissue heart samples and skin fibroblasts provide a method to visualize the pathophysiology of disease-associated mutations; however, they are not easy to acquire. Currently, no specific therapy exists for patients with LMNA-related DCM. This has encouraged researchers to establish both mice and cellular models in an effort to elucidate the mechanisms leading to the disease phenotypes. Unraveling the molecular mechanisms might provide insights into the pathophysiology of this disease which could be translated into novel therapy in the future.

A Lmna null mouse based on genetrap technology has been developed [24]. The mouse is characterized by postnatal maturation defects of cardiac, muscle, and adipose tissues. Premature death occurred by $2-3$ weeks of age. However, in this study, age matched heterozygous mice were indistinguishable from wild-type mice [24]. Only 1 study reported $L m n a^{+/-}$mice with $50 \%$ of normal cardiac lamin $\mathrm{A} / \mathrm{C}$ levels and displaying cardiac abnormalities [25]. The Lmna ${ }^{\mathrm{H} 222 \mathrm{P} / \mathrm{H} 222 \mathrm{P}}$ mice harbouring the EDMD mutation developed muscular dystrophy and DCM with atrio-ventricular conduction defect at adulthood and died by 13 months of age [26]. Male Lmna ${ }^{\mathrm{H} 222 \mathrm{P} / \mathrm{H} 222 \mathrm{P}}$ mice developed significant left ventricular dilatation and by 16 weeks of age had decreased ejection fraction [26]. In another study, Lmna ${ }^{\mathrm{N} 195 \mathrm{~K} / \mathrm{N} 195 \mathrm{~K}}$ mice harboring a DCM with conduction system disease mutation, died at an early age due to arrhythmia. Surprisingly, both $\mathrm{Lmna}{ }^{\mathrm{H} 222 \mathrm{P} /+}$ and $\mathrm{Lmna}^{\mathrm{N} 195 \mathrm{~K} /+}$ mice were found to have a phenotype and life expectancy similar to the wild-type [26, 27]. Cells derived from both $\mathrm{Lmna}^{-/-}$and Lmna ${ }^{\mathrm{N} 195 \mathrm{~K} / \mathrm{N} 195 \mathrm{~K}}$ mice were observed to have damaged and misshapen nuclei, showed increased fragility under mechanical strain and impaired gene transcription [27-30].

Lamin A/C is found in almost all cells except in certain differentiated cells of hematopoietic origins [31]. Cellular models have shown that lamin $\mathrm{A}$ and $\mathrm{C}$ proteins are found distributed together in a homogeneous meshwork. However, wild type lamin A transfected alone has consistently shown to localize to the inner nuclear lamina with some nucleoplasmic localization. Conversely, lamin $\mathrm{C}$ has been shown to localize as intranuclear aggregate $[18,32-36]$. Intranuclear lamin $\mathrm{C}$ has shown to be more mobile than intranuclear lamin A [36, 37]. Likewise, the lamin $\mathrm{C}$ only mouse model expressed lamin $\mathrm{C}$ at the inner nuclear lamina as established in wild type cells [35]; thus indicating the existence of compensatory mechanisms. Pugh et al. [32] studied the incorporation of the lamin A and C in Swiss 3T3 cells and found that the incorporation of lamin $\mathrm{C}$ into the lamina was made possible by lamin $\mathrm{A}$.

In an attempt to identify deregulation in striated muscle specific laminopathies including DCM and EDMD, researches have been focused on skeletal muscle differentiations. Lamin $\mathrm{A}$ and $\mathrm{C}$ play a pivotal role in myoblast differentiation. In vitro, cells expressing disease-associated LMNA mutations displayed an inhibition of myoblast differentiation [38] (F. Tesson personal communication) and myoblasts lacking lamin $\mathrm{A}$ and $\mathrm{C}$ expression showed decreased differentiation potential with downregulation of $\mathrm{MyoD}$ and $\mathrm{pRb}$ and upregulation of Myf5 [39]. These studies suggest that disruption of lamin A and C may weaken contractile tissues such as skeletal and cardiac muscle.

Lamin $\mathrm{A}$ and $\mathrm{C}$ also play a role in the regulation of signaling cascades such as the Sumo pathway. Sumo pathway regulates a wide range of cellular processes through the attachment of small ubiquitin-related modifier (sumo) to various substrates. Sumo1 was found to be mislocalized in the presence of lamin $\mathrm{A}$ and $\mathrm{C}$ mutants both in vitro (C2C12 and Cos7 cells) and in vivo (primary myoblasts and myopathic muscle tissue from 
the Lmna ${ }^{\mathrm{H} 222 \mathrm{P} / \mathrm{H} 222 \mathrm{P}}$ mice) $[18,40]$. In cell models, trapping of sumo1 correlated with an increased steady-state level of sumoylation. Ubc9, the E2 conjugating enzyme of the Sumo pathway was also mislocalized to the mutant aggregates [40]. Lamin A has been shown to be covalently modified by Sumo 2 and 3 [41]. The disruption of a critical post-translational modifying process has the potential to affect the post-translational regulation of tissue-specific sumoylated proteins which may lead to the tissue-specific symptoms observed in patients with various laminopathies [40].

Recent studies using induced pluripotent stem cells derived cardiomyocytes (iPSCS-CMs) from DCM patients with LMNA mutations showed accelerated nuclear senescence and apoptosis under electrical stimulation. This study also showed that activation of stress response MEK1/ERK1/2 pathway contributes to increased apoptosis in LMNA $^{\text {R225X/WT }}$ dermal fibroblasts after electrical stimulation [42]. Moreover, this apoptotic effect could be attenuated by pharmacological blockade of the MEK1/ERK1/2 pathway. Study of gene expression profile showed that mouse models of laminopathies also displayed ERK pathway activation in heart muscle [43, 44]. Importantly, the pharmacological blockade of the ERK1/2 pathway prevented the development of DCM in this model [45]. These studies have shed new light on MEK1 pathway as a potential therapeutic target in LMNA-associated DCM.

\section{Conclusion: The mechanistic hypotheses}

Until now, 3 main hypotheses have been proposed to explain the mechanism of pathogenesis of laminopathies: the structural, the gene expression and the toxicity hypotheses. The structural hypothesis states that mutations within lamin $\mathrm{A} / \mathrm{C}$ lead to disorganization of the proteinaceous meshwork, instability of the nuclear envelope and disorganization of chromatin, which in turn leads to the overall inability of the cell to properly function in contracting tissue environment such as striated muscles [46, 47]. Building on this hypothesis, recent studies identified repetitive disruptions of the nuclear envelope in the presence of lamin $\mathrm{A} / \mathrm{C}$ mutations $[17,48]$. These disruptions impaired protein distribution into cell compartments. Translocations of large amounts of protein into the cytoplasm could trigger aggresome formation or even induce cell apoptosis [48]. On the other hand, translocation of transcription factors into the cytoplasm might impair gene expression. The gene expression hypothesis is based on the regulatory role of lamin $\mathrm{A} / \mathrm{C}$ in chromatin organization and DNA transcription. Mutated lamins might disrupt the protein meshwork through their interaction with other proteins of the nuclear envelope which may lead to epigenetic changes in the chromatin, which may then in turn disrupt various complex signaling pathways [49]. Lastly, the cell toxicity hypothesis proposes that mutated prelamin A may accumulate within patients' nuclei to the point that they might become toxic to the cell and lead to development of the disease [50]. These hypotheses are likely to be not mutually exclusive and combining them might allow describing the mechanisms underlying the initiation and/or the development of laminopathies. Ultimately, a better understanding of the pathogenesis of the disease may suggest novel strategies targeting the underlying molecular defects.

\section{Conflict of interest: none declared}

\section{References}

1. Elliott P, Andersson B, Arbustini E et al. [Classification of the cardiomyopathies]. Kardiol Pol, 2008, 66: 533-540.

2. Maron BJ, Towbin JA, Thiene G et al. Contemporary definitions and classification of the cardiomyopathies: An American Heart Association Scientific Statement from the Council on Clinical Cardiology, Heart Failure and Transplantation Committee; Quality of Care and Outcomes Research and Functional Genomics and Translational Biology Interdisciplinary Working Groups; and Council on Epidemiology and Prevention. Circulation, 2006; 113: 1807-1816.

3. Ghosh N, Haddad H. Recent progress in the genetics of cardiomyopathy and its role in the clinical evaluation of patients with cardiomyopathy. Curr Opin Cardiol, 2011; 26: 155-164.

4. Taylor MR, Carniel E, Mestroni L. Cardiomyopathy, familial dilated. Orphanet J Rare Dis, 2006; 1: 27.

5. Hershberger RE, Siegfried JD. Update 2011: Clinical and genetic issues in familial dilated cardiomyopathy. J Am Coll Cardiol, 2011; 57: 1641-1649.

6. Pasotti M, Klersy C, Pilotto A et al. Long-term outcome and risk stratification in dilated cardiolaminopathies. J Am Coll Cardiol, 2008; 52: 1250-1260.

7. Mangin L, Charron $\mathrm{Ph}$, Tesson $\mathrm{F}$ et al. Familial dilated cardiomyopathy: Clinical features in French families. J Heart Failure, 1999; 1: 353-361.

8. Mestroni L, Rocco C, Gregori D et al.; Heart Muscle Disease Study Group. Familial dilated cardiomyopathy: Evidence for genetic and phenotypic heterogeneity. J Am Coll Cardiol, 1999; 34: 181-190.

9. Brodsky GL, Muntoni F, Miocic S, Sinagra G, Sewry C, Mestroni L. Lamin A/C gene mutation associated with dilated cardiomyopathy with variable skeletal muscle involvement. Circulation, 2000; 101: 473-476.

10. Bonne G, Di Barletta MR, Varnous S et al. Mutations in the gene encoding lamin $\mathrm{A} / \mathrm{C}$ cause autosomal dominant Emery-Dreifuss muscular dystrophy. Nat Genet, 1999; 21: 285-288.

11. Fatkin D, MacRae C, Sasaki T et al. Missense mutations in the rod domain of the lamin $\mathrm{A} / \mathrm{C}$ gene as causes of dilated cardiomyopathy and conduction-system disease. N Engl J Med, 1999; 341: 1715-1724. 
12. Bertrand AT, Chikhaoui K, Yaou RB, Bonne G. Clinical and genetic heterogeneity in laminopathies. Biochem Soc Trans, 2011; 39: 1687-1692.

13. Szeverenyi I, Cassidy AJ, Chung $\mathrm{CW}$ et al. The Human Intermediate Filament Database: Comprehensive information on a gene family involved in many human diseases. Hum Mutat, 2008; 29: 351-360.

14. Broers JL, Ramaekers FC, Bonne G, Yaou RB, Hutchison CJ. Nuclear lamins: Laminopathies and their role in premature ageing. Physiol Rev, 2006; 86: 967-1008.

15. Lin F, Worman HJ. Structural organization of the human gene encoding nuclear lamin A and nuclear lamin C. J Biol Chem, 1993; 268: 16321-16326.

16. Bertrand AT, Chikhaoui K, Yaou RB, Bonne G. Clinical and genetic heterogeneity in laminopathies. Biochem Soc Trans, 2011; 39: 1687-1692.

17. Gupta P, Bilinska ZT, Sylvius N et al. Genetic and ultrastructural studies in dilated cardiomyopathy patients: A large deletion in the lamin $\mathrm{A} / \mathrm{C}$ gene is associated with cardiomyocyte nuclear envelope disruption. Basic Res Cardiol, 2010; 105: 365-377.

18. Sylvius N, Bilinska ZT, Veinot JP et al. In vivo and in vitro examination of the functional significances of novel lamin gene mutations in heart failure patients. J Med Genet, 2005; 42: 639-647.

19. Arbustini E, Pilotto A, Repetto A et al. Autosomal dominant dilated cardiomyopathy with atrioventricular block: A lamin A/C defect-related disease. J Am Coll Cardiol, 2002; 39: 981-990.

20. Fidzianska A, Bilinska ZT, Tesson F et al. Obliteration of cardiomyocyte nuclear architecture in a patient with LMNA gene mutation. J Neurol Sci, 2008; 271: 91-96.

21. Muchir A, Worman HJ. The nuclear envelope and human disease. Physiology (Bethesda), 2004; 19: 309-314

22. Favreau C, Dubosclard E, Ostlund C et al. Expression of lamin A mutated in the carboxyl-terminal tail generates an aberrant nuclear phenotype similar to that observed in cells from patients with Dunnigan-type partial lipodystrophy and Emery-Dreifuss muscular dystrophy. Exp Cell Res, 2003; 282: 14-23.

23. Muchir A, Medioni J, Laluc M et al. Nuclear envelope alterations in fibroblasts from patients with muscular dystrophy, cardiomyopathy, and partial lipodystrophy carrying lamin $\mathrm{A} / \mathrm{C}$ gene mutations. Muscle Nerve, 2004; 30: 444-450.

24. Kubben N, Voncken JW, Konings G et al. Post-natal myogenic and adipogenic developmental: Defects and metabolic impairment upon loss of A-type lamins. Nucleus, 2011; 2: 195-207.

25. Wolf CM, Wang L, Alcalai R et al. Lamin A/C haploinsufficiency causes dilated cardiomyopathy and apoptosis-triggered cardiac conduction system disease. J Mol Cell Cardiol, 2008; 44: 293-303.

26. Arimura T, Helbling-Leclerc A, Massart $\mathrm{C}$ et al. Mouse model carrying H222P-Lmna mutation develops muscular dystrophy and dilated cardiomyopathy similar to human striated muscle laminopathies. Hum Mol Genet, 2005; 14: 155-169.

27. Mounkes LC, Kozlov SV, Rottman JN, Stewart CL. Expression of an LMNA-N195K variant of A-type lamins results in cardiac conduction defects and death in mice. Hum Mol Genet, 2005; 14: 2167-2180.

28. Sullivan T, Escalante-Alcalde D, Bhatt $\mathrm{H}$ et al. Loss of A-type lamin expression compromises nuclear envelope integrity leading to muscular dystrophy. J Cell Biol, 1999; 147: 913-920.

29. Lammerding J, Schulze PC, Takahashi T et al. Lamin A/C deficiency causes defective nuclear mechanics and mechanotransduction. J Clin Invest, 2004; 113: 370-378.

30. Nikolova V, Leimena C, McMahon AC et al. Defects in nuclear structure and function promote dilated cardiomyopathy in lamin A/C-deficient mice. J Clin Invest, 2004; 113: 357-369.

31. Broers JL, Machiels BM, Kujipers HJ et al. A-and B-type lamins are differentially expressed in normal human tissues. Histochem Cell Biol, 1997; 107: 505-517.
32. Pugh GE, Coates PJ, Lane EB, Raymond Y, Quinlan RA. Distinct nuclear assembly pathways for lamins $\mathrm{A}$ and $\mathrm{C}$ lead to their increase during quiescence in Swiss 3T3 cells. J Cell Sci, 1997; 110 (Part 19): 2483-2493.

33. Raharjo WH, Enarson P, Sullivan T, Stewart CL, Burke B. Nuclear envelope defects associated with LMNA mutations cause dilated cardiomyopathy and Emery-Dreifuss muscular dystrophy. J Cell Sci, 2001; 114: 4447-4457.

34. Vaughan A, Alvarez-Reyes M, Bridger JM et al. Both emerin and lamin $\mathrm{C}$ depend on lamin $\mathrm{A}$ for localization at the nuclear envelope. J Cell Sci, 2001; 114: 2577-2590.

35. Fong LG, Ng JK, Lammerding J et al. Prelamin A and lamin A appear to be dispensable in the nuclear lamina. J Clin Invest, 2006; 116: 743-752.

36. Sylvius N, Hathaway A, Boudreau E et al. Specific contribution of lamin $\mathrm{A}$ and lamin $\mathrm{C}$ in the development of laminopathies. Exp Cell Res, 2008; 314: 2362-2375.

37. Broers JL, Kuijpers HJ, Ostlund C, Worman HJ, Endert J, Ramaekers FC. Both lamin A and lamin C mutations cause lamina instability as well as loss of internal nuclear lamin organization. Exp Cell Res, 2005; 304: 582-592.

38. Favreau C, Higuet D, Courvalin JC, Buendia B. Expression of a mutant lamin A that causes Emery-Dreifuss muscular dystrophy inhibits in vitro differentiation of $\mathrm{C} 2 \mathrm{C} 12$ myoblasts. Mol Cell Biol, 2004; 24: 1481-1492.

39. Frock RL, Kudlow BA, Evans AM, Jameson SA, Hauschka SD, Kennedy BK. Lamin A/C and emerin are critical for skeletal muscle satellite cell differentiation. Genes Dev, 2006; 20: 486-500.

40. Boudreau E, Labib S, Bertrand AT et al. Lamin A/C mutants disturb sumo1 localization and sumoylation in vitro and in vivo. PLoS One, 2012; 7: e45918.

41. Zhang YQ, Sarge KD. Sumoylation regulates lamin A function and is lost in lamin A mutants associated with familial cardiomyopathies. J Cell Biol, 2008; 182: 35-39.

42. Siu CW, Lee YK, Ho JC et al. Modeling of lamin A/C mutation premature cardiac aging using patient-specific induced pluripotent stem cells. Aging (Albany NY), 2012; 4: 803-822.

43. Muchir A, Pavlidis P, Decostre V et al. Activation of MAPK pathways links LMNA mutations to cardiomyopathy in Emery-Dreifuss muscular dystrophy. J Clin Invest, 2007; 117: 1282$-1293$.

44. Muchir A, Pavlidis P, Bonne G, Hayashi YK, Worman HJ. Activation of MAPK in hearts of EMD null mice: Similarities between mouse models of X-linked and autosomal dominant Emery Dreifuss muscular dystrophy. Hum Mol Genet, 2007; 16: 1884-1895.

45. Muchir A, Shan J, Bonne G, Lehnart SE, Worman HJ. Inhibition of extracellular signal-regulated kinase signaling to prevent cardiomyopathy caused by mutation in the gene encoding A-type lamins. Hum Mol Genet, 2009; 18: 241-247.

46. Broers JL, Peeters EA, Kuijpers HJ et al. Decreased mechanical stiffness in LMNA-/- cells is caused by defective nucleo-cytoskeletal integrity: implications for the development of laminopathies. Hum Mol Genet, 2004; 13: 2567-2580.

47. Fidzianska A, Hausmanowa-Petrusewicz I. Architectural abnormalities in muscle nuclei. Ultrastructural differences between $\mathrm{X}$-linked and autosomal dominant forms of EDMD. J Neurol Sci, 2003; 210: 47-51.

48. De Vos WH, Houben F, Kamps M et al. Repetitive disruptions of the nuclear envelope invoke temporary loss of cellular compartmentalization in laminopathies. Hum Mol Genet, 2011; 20: 4175-4186.

49. Maraldi NM, Capanni C, Cenni V, Fini M, Lattanzi G. Laminopathies and lamin-associated signaling pathways. J Cell Biochem, 2011; 112: 979-992.

50. Navarro CL, Cau P, Levy N. Molecular bases of progeroid syndromes. Hum Mol Genet, 2006, 15 Spec. No. 2: R151-R161. 
Table 1. LMNA mutations associated with dilated cardiomyopathy from four databases - Human Intermediate Filament Database [13] (database updated 2014/01/15), Leiden Muscular Dystrophy website (www.dmd.nl; database updated 2014/01/05), HGMD ${ }^{\circledR}$ Professional 2014.4 (database updated 2014/02/02) and the Universal Mutation Database (www.umd.be/LMNA/).

\begin{tabular}{|c|c|c|c|c|}
\hline Nucleotide change & Protein change & DCM phenotype range & Domain & Reference* \\
\hline $\begin{array}{l}\text { 674bp deletion incl. } \\
\text { start codon }\end{array}$ & Deletion of $5^{\prime}$ part & $\begin{array}{c}1^{\circ} \mathrm{AVB}, \mathrm{LBBB}, \mathrm{AF}, \mathrm{PVB}, \\
\mathrm{VT}, \mathrm{HF}, \mathrm{SCD}\end{array}$ & Head & [1] \\
\hline c. $16 \mathrm{C}>\mathrm{T}$ & p.GIn6X & $\begin{array}{r}1^{\circ}-2^{\circ} \mathrm{AVB}, \mathrm{AF}, \mathrm{AFL}, \\
\mathrm{PAB}, \mathrm{PVB}, \mathrm{VT}, \mathrm{HF}, \mathrm{SC} D\end{array}$ & Head & [2] \\
\hline c.28_29insA & p.Thr10AsnfsX31 & $1^{\circ}-2^{\circ} \mathrm{AVB}, \mathrm{AF}, \mathrm{PVB}, \mathrm{MD}$ & Head & [3] \\
\hline c.31delC & p.Arg11AlafsX85 & $\left(2^{\circ}\right) A V B$ & Head & {$[4,5]$} \\
\hline c.46_49dup & p.Ser18GInfsX24 & AVB & Head & [6] \\
\hline c.48_51dupCAGC & p.Ser18GInfsX23 & $\mathrm{n} / \mathrm{a}$ & Head & [6] \\
\hline c. $65 \overline{\mathrm{C}}>\mathrm{T}$ & p.Ser22Leu & $\mathrm{PVB}, \mathrm{HF}$ & Head & [7] \\
\hline c. $73 \mathrm{C}>\mathrm{T}$ & p.Arg25Cys & $C A, A F, P V B, M D, H F$ & Head & [8] \\
\hline c. $73 C>G$ & p.Arg25Gly & $\begin{array}{c}1 \% 3^{\circ} \text { AVB, TC, AF, PVB, PAB, } \\
\text { LGMD, HF }\end{array}$ & Head & [9] \\
\hline c. $78 \mathrm{C}>\mathrm{T}$ & p.lle26lle & $\mathrm{HF}, \mathrm{LBBB}, \mathrm{AF}$ & Head & [10] \\
\hline c. $82 \mathrm{C}>\mathrm{T}$ & p.Arg28Trp & AVB, AF, PM, FPLD, HF & Head & {$[4,11]$} \\
\hline c.94_96delAAG & p.Lys32del & LAFB, CA, AF, EDMD & Head & {$[12,13]$} \\
\hline c. $99 \mathrm{G}>\mathrm{T}$ & p.Glu33Asp & $\begin{array}{c}1^{\circ} \mathrm{AVB}, \mathrm{Br}, \mathrm{AF}, \mathrm{CA}, \mathrm{CMT} 2, \mathrm{MD}, \\
\text { leuconychia }\end{array}$ & Head & [14] \\
\hline c. $106 \mathrm{C}>\mathrm{T}$ & p.Gln36X & $1^{\circ}-2^{\circ} \mathrm{AVB}, \mathrm{VT}$ & Coil $1 \mathrm{~A}$ & [15] \\
\hline c. $134 \mathrm{~A}>\mathrm{G}$ & p.Tyr45Cys & $A F, A F L, M D$ & Coil $1 \mathrm{~A}$ & [16] \\
\hline c. $154 C>G$ & p.Leu52Val & $\mathrm{n} / \mathrm{a}$ & Coil 1A & [17] \\
\hline c. $155 T>C$ & p.Leu52Pro & AVB, LBBB, RBBB, AF, PVB, HF & Coil $1 \mathrm{~A}$ & [18] \\
\hline c. $158 \mathrm{~A}>\mathrm{T}$ & p.Glu53Val & $\mathrm{AVB}, \mathrm{AF}, \mathrm{HF}$ & Coil $1 \mathrm{~A}$ & [19] \\
\hline c. $165 \mathrm{delC}$ & p.Asn56Thrfs X40 & $2^{\circ} \mathrm{AVB}, \mathrm{HF}$ & Coil $1 \mathrm{~A}$ & [5] \\
\hline c. $169 \mathrm{G}>\mathrm{C}$ & p.Ala57Pro & $\begin{array}{l}\text { Atypical WS, hypogonadism, } \\
\text { sloping shoulders }\end{array}$ & Coil $1 \mathrm{~A}$ & [20] \\
\hline c. $176 \mathrm{~T}>\mathrm{G}$ & p.Leu59Arg & $\begin{array}{l}\text { CCD, hypogonadism, } \\
\text { ovarian failure, MAD }\end{array}$ & Coil $1 \mathrm{~A}$ & {$[21,22]$} \\
\hline c. $178 \mathrm{C}>\mathrm{G}$ & p.Arg60Gly & $\begin{array}{c}1 \% 3^{\circ} \text { AVB, LBBB, Br, } \\
\text { AF, VA, DM, FPLD, PN, } \\
\text { HF, SCD }\end{array}$ & Coil $1 \mathrm{~A}$ & [23-26] \\
\hline c. $184 C>G$ & p.Arg62Gly & $\mathrm{CCD}, 1^{\circ} \mathrm{AVB}, \mathrm{AF}, \mathrm{PM}, \mathrm{FPLD}, \mathrm{HF}$ & Coil $1 \mathrm{~A}$ & {$[11,26]$} \\
\hline c.203_208delAGGTGG & p.Glu68_Val69del & $3^{\circ}$ AVB, EDMD(2) & Coil 1A & {$[4,27]$} \\
\hline c. $215 \mathrm{G}>\mathrm{T}$ & p.Arg72Leu & $\mathrm{n} / \mathrm{a}$ & Linker 1 & [17] \\
\hline c. $232 A>G$ & p.Lys78Glu & $1^{\circ} \mathrm{AVB}, \mathrm{VT}, \mathrm{ICD}$ & Linker 1 & {$[96]$} \\
\hline c. $244 \mathrm{G}>\mathrm{A}$ & p.Glu82Lys & $\begin{array}{c}1^{\circ}-3^{\circ} \text { AVB, LBBB, AF, VF, } \\
\text { SVT, HF }\end{array}$ & Coil 1B & {$[28,29]$} \\
\hline c. $254 \mathrm{~T}>\mathrm{G}$ & p.Leu85Arg & CCD, PM, AF, HF, SCD & Coil 1B & [23] \\
\hline c. $266 \mathrm{G}>\mathrm{T}$ & p.Arg89Leu & $\begin{array}{l}1^{\circ}-3^{\circ} \mathrm{AVB}, \mathrm{AF}, \mathrm{VT}, \mathrm{MA}, \\
\mathrm{HF} / \mathrm{AVB}, \mathrm{AF}, \mathrm{VT}, \mathrm{EDMD}, \mathrm{HF}\end{array}$ & Coil 1B & {$[4,5,30-32]$} \\
\hline c. $274 \mathrm{C}>\mathrm{T}$ & p.Leu92Phe & LBBB, AF, PVB, HF & Coil 1B & {$[33,34]$} \\
\hline c. $289 A>G$ & p.Lys97Glu & $1^{\circ} / 3^{\circ}$ AVB, LBBB, PVB, HF & Coil 1B & {$[4,5,35]$} \\
\hline c. $302 \mathrm{G}>\mathrm{C}$ & p.Arg101Pro & $\begin{array}{c}\text { AF, HF / AVB, AF, PVB, } \\
\text { VT, LGMD }\end{array}$ & Coil 1B & [31] / [94] \\
\hline c. $331 \mathrm{G}>\mathrm{T}$ & p.Glu111X & $3^{\circ}$ AVB, PVB, HF & Coil 1B & {$[4,5,35]$} \\
\hline c.348_349insG & p.Lys117GlufsX10 & AVB, AF, SCD & Coil 1B & [36] \\
\hline c. $356+1 \mathrm{G}>\mathrm{T}$ & $\mathrm{n} / \mathrm{a}$ & $\mathrm{n} / \mathrm{a}$ & Coil 1B & [37] \\
\hline c.357-1G >T & $\mathrm{n} / \mathrm{a}$ & LBBB, AF, PVB, VT, VF, HF & Coil 1B & {$[4,31,34]$} \\
\hline c.357C $>\mathrm{T}$ & p.Arg119Arg & CCD, PM, LGMD, HF & Coil 1B & [16] \\
\hline c.367_369del & p.Lys123del & $\begin{array}{c}1^{\circ}-3^{\circ} \text { AVB, LBBB, PVB, } \\
\text { VT, VF, MP, SCD }\end{array}$ & Coil 1B & [38] \\
\hline c.380_381ins24bp & $\begin{array}{l}\text { p.lle128 Ala129ins- } \\
\text { ArgValThrLeulle- } \\
\text { SerSerArg }\end{array}$ & CCD & Coil 1B & [34] \\
\hline c.384ins24 & p.lle128delinslleSer & $\mathrm{n} / \mathrm{a}$ & Coil 1B & [33] \\
\hline
\end{tabular}




\begin{tabular}{|c|c|c|c|c|}
\hline Nucleotide change & Protein change & DCM phenotype range & Domain & Reference* \\
\hline c. $394 G>C$ & p.Ala132Pro & $1^{\circ} \mathrm{AVB}, \mathrm{Br}, \mathrm{AF}, \mathrm{HF}$ & Coil 1B & [39] \\
\hline c. $398 \mathrm{G}>\mathrm{T}$ & p.Arg133Leu & CCD, LD & Head & [40] \\
\hline c.405_425dup & p.Asn142delinsLysLys & $1^{\circ} \mathrm{AVB}, \mathrm{AF}, \mathrm{VT}, \mathrm{PVB}$ & Coil 1B & [41] \\
\hline $\begin{array}{l}\text { c. } 425 \text { 426insGGCACTG } \\
\text { GAGGCTCTGCTGAA }\end{array}$ & $\begin{array}{l}\text { p.Leu141_Asn142insLys- } \\
\text { AspLeuĀspAlaLeuLeu }\end{array}$ & $1^{\circ} \mathrm{AVB}, \mathrm{AF}, \mathrm{PVB}, \mathrm{VT}, \mathrm{HF}$ & Coil 1B & [41] \\
\hline c. $427 \mathrm{~T}>\mathrm{C}$ & p.Ser143Pro & $\begin{array}{c}1^{\circ}-3^{\circ} \text { AVB, LBBB, LAFB, SSS, } \\
\text { Br, AF, PVB, VF, VT, HF }\end{array}$ & Coil 1B & {$[39,42]$} \\
\hline c. $481 \mathrm{G}>\mathrm{A}$ & p.Glu161Lys & $\begin{array}{l}\text { AVB, LBBB, Br, AF, PVB, } \\
\text { LAFB, VT, HF }\end{array}$ & Coil 1B & $\begin{array}{l}{[4,3,19,} \\
34,43]\end{array}$ \\
\hline c. $497 \mathrm{G}>\mathrm{C}$ & p.Arg166Pro & AVB, LBBB, AF, VT, HF & Coil 1B & {$[31,44]$} \\
\hline c. $514-1 \mathrm{G}>\mathrm{A}$ & $\mathrm{n} / \mathrm{a}$ & VT, VF & Coil 1B & [4] \\
\hline c. $548 \mathrm{~T}>\mathrm{C}$ & p.Leu183Pro & AVB & Coil 1B & {$[4]$} \\
\hline c. $556 \mathrm{G}>\mathrm{A}$ & p.Glu186Lys & $\mathrm{CCD}, \mathrm{HF}$ & Coil 1B & [19] \\
\hline c. $565 \mathrm{C}>\mathrm{T}$ & p.Arg189Trp & PVB & Coil 1B & [45] \\
\hline c. $568 \mathrm{C}>\mathrm{T}$ & p.Arg190Trp & $\begin{array}{c}1^{\circ} \mathrm{AVB}, \mathrm{RBBB}, \mathrm{Br}, \mathrm{LAFB}, \mathrm{AF}, \\
\text { AFL, PVB, HF }\end{array}$ & Coil 1B & $\begin{array}{l}{[4,5,7,19,35,} \\
\quad 39,46,47]\end{array}$ \\
\hline c. $569 \mathrm{G}>\mathrm{A}$ & p.Arg190GIn & $\begin{array}{c}1^{\circ}-3^{\circ} \mathrm{AVB}, \mathrm{Br}, \mathrm{AF}, \mathrm{AFL}, \\
\text { VT, HF }\end{array}$ & Coil 1B & {$[31,43]$} \\
\hline c. $575 \mathrm{~A}>\mathrm{G}$ & p.Asp192Gly & $1^{\circ} \mathrm{AVB}, \mathrm{LAFB}, \mathrm{HF}$ & Coil 1B & {$[46,48,49]$} \\
\hline c. $575 \mathrm{~A}>\mathrm{T}$ & p.Asp192Val & LBBB, FLPD, HF & Coil 1B & {$[26]$} \\
\hline c. $585 \mathrm{C}>\mathrm{G}$ & p.Asn 195Lys & $1^{\circ}-3^{\circ} \mathrm{AVB}, \mathrm{Br}, \mathrm{AF}, \mathrm{HF}, \mathrm{SCD}$ & Coil 1B & {$[8,23]$} \\
\hline c. $585 \mathrm{C}>\mathrm{A}$ & p.Asn195Lys & $1^{\circ}, 3^{\circ} \mathrm{AVB}, \mathrm{AF}, \mathrm{HF}, \mathrm{SCD}$ & Coil 1B & [8] \\
\hline c. $607 \mathrm{G}>\mathrm{A}$ & p.Glu203Lys & $1^{\circ} \mathrm{AVB}, \mathrm{LBBB}, \mathrm{RBBB}, \mathrm{HF}$ & Coil 1B & {$[50]$} \\
\hline c. $608 \mathrm{~A}>\mathrm{G}$ & p.Glu203Gly & $1^{\circ}-2^{\circ} \mathrm{AVB}, \mathrm{AF}, \mathrm{HF}, \mathrm{SCD}$ & Coil 1B & [23] \\
\hline c. $608 \mathrm{~A}>\mathrm{T}$ & p.Glu203Val & $2^{\circ} \mathrm{AVB}, \mathrm{Br}, \mathrm{SWMA}, \mathrm{HF}$ & Coil 1B & {$[23,43]$} \\
\hline c.622_624delAAG & p.Lys208del & $1^{\circ} \mathrm{AVB}, \mathrm{PVB}, \mathrm{VT}, \mathrm{LGMD}$ & Coil 1B & [8] \\
\hline c. $629 \mathrm{~T}>\mathrm{G}$ & p.lle210Ser & $\mathrm{AF}, \mathrm{HF}$ & Coil 1B & {$[31,51]$} \\
\hline c. $640-10 A>G$ & $\mathrm{n} / \mathrm{a}$ & $\begin{array}{c}1^{\circ}-3^{\circ} \text { AVB, LBBB, RBBB, } \\
\text { AF, VT, HF }\end{array}$ & Coil 1B & {$[52]$} \\
\hline c.644T >C & p.Leu215Pro & $\begin{array}{l}1^{\circ}-2^{\circ} \text { AVB, SSS, Br, LBBB, AF, } \\
\text { AFL, SVT, VT, PVB, SCD, HF }\end{array}$ & Coil 1B & [53] \\
\hline c. $656 \mathrm{~A}>\mathrm{C}$ & p.Lys219Thr & $\left(3^{\circ}\right) A V B$ & Linker 2 & {$[4,43]$} \\
\hline c. $657 \mathrm{G}>\mathrm{C}$ & p.Lys219Asn & $1^{\circ} \mathrm{AVB}, \mathrm{VT}, \mathrm{PM}$ & Linker 2 & {$[54]$} \\
\hline c. $673 \mathrm{C}>\mathrm{T}$ & p.Arg225X & $\left(1^{\circ}\right) \mathrm{AVB}, \mathrm{Br}, \mathrm{AF}, \mathrm{LVE}, \mathrm{PVB}, \mathrm{SCD}$ & Linker 2 & {$[8,44,50]$} \\
\hline c. $676 \mathrm{C}>\mathrm{G}$ & p.Leu226Val & $\mathrm{n} / \mathrm{a}$ & Linker 2 & [37] \\
\hline c. $694 \mathrm{G}>\mathrm{C}$ & p.Gly232Arg & AVB, RBBB, PVB, EDMD & Linker 2 & [18] \\
\hline c. $700 \mathrm{C}>\mathrm{T}$ & p.GIn234X & $2^{\circ} \mathrm{AVB}, \mathrm{Br}, \mathrm{HF}$ & Linker 2 & [31] \\
\hline c. $736 \mathrm{C}>\mathrm{T}$ & p.GIn246X & AVB & Coil 2 & {$[4]$} \\
\hline c.746G $>A$ & p.Arg249GIn & AVB, PVB, EDMD or LGMD(1B) & Coil 2 & [18] \\
\hline c.767T>G & p.Val256IGly & AVB & Coil 2 & {$[55]$} \\
\hline c. $775 \mathrm{~T}>\mathrm{C}$ & p.Tyr259His & AVB, AF, VT & Coil 2 & {$[44]$} \\
\hline c. $780 \mathrm{G}>\mathrm{C}$ & p.Lys260Asn & AVB, SSS, ASS, HF & Coil 2 & {$[4,56]$} \\
\hline c.781_783del3ins18 & p.Lys261delins 6 & $\begin{array}{l}\text { AVB, LBBB, AF, } \\
\text { PVC, } \operatorname{EDMD(2)}\end{array}$ & Coil 2 & [18] \\
\hline c.799T>C & p.Tyr267His & CCD, SCD & Coil 2 & [57] \\
\hline c. $800 A>G$ & p.Tyr267Cys & AVB, TC, EDMD(2) & Coil 2 & {$[4,58]$} \\
\hline c. $810 \mathrm{G}>\mathrm{A}$ & p.Lys270Lys & CCD, AF, PM, EDMD, LGMD & Coil 2 & [16] \\
\hline c. $811-3 C>T$ & $\mathrm{n} / \mathrm{a}$ & $\mathrm{n} / \mathrm{a}$ & Coil 2 & [37] \\
\hline c.812T >C & p.Leu271Pro & AF, Br, SVT, VT, EDMD, HF & Coil 2 & {$[16,59]$} \\
\hline $\begin{array}{l}\text { c.815 818delACA } \\
\text { AinsCCAGAC }\end{array}$ & p.Asp272AlafsX208 & AVB, AF, VT & Coil 2 & [44] \\
\hline c. $832 \mathrm{G}>\mathrm{A}$ & p.Ala278Thr & AVB, AF, VF, SVT, PM, PVB & Coil 2 & {$[60]$} \\
\hline c.855delG & p.Ala287LeufsX191 & AF, ASS, LGMD & Coil 2 & [58] \\
\hline c.859insC & p.Ala287fs & $\mathrm{AF}$ & Coil 2 & [34] \\
\hline c.883T $>C$ & p.Ser295Pro & $\mathrm{CCD}, \mathrm{MD}$ & Coil 2 & [16] \\
\hline c.906_907delCT & p.Ser303CysfsX27 & $\begin{array}{c}\text { AVB, PM, HF, Br, PAB, PVB, } \\
\text { CCD, SVA, AF, SCD }\end{array}$ & Coil 2 & {$[95]$} \\
\hline
\end{tabular}




\begin{tabular}{|c|c|c|c|c|}
\hline Nucleotide change & Protein change & DCM phenotype range & Domain & Reference* \\
\hline c.908_909delCT & p.Ser303CysfsX26 & $\begin{array}{c}1^{\circ} \mathrm{AVB}, \mathrm{Br}, \mathrm{SSS}, \mathrm{AF} / 1^{\circ}-3^{\circ} \mathrm{AVB}, \\
\text { AF, PVB, VT, LGMD, HF }\end{array}$ & Coil 2 & {$[61,62]$} \\
\hline c. $936 \mathrm{G}>\mathrm{C}$ & p.Gln312His & $\mathrm{AVB}, \mathrm{AF}, \mathrm{CCD}, \mathrm{HF}$ & Coil 2 & [63] \\
\hline c. $976 \mathrm{~T}>\mathrm{A}$ & p.Ser326Thr & $\begin{array}{c}\text { VT, AVB, CCD, XL-EDMD, } \\
\text { MP, PM }\end{array}$ & Coil 2 & [97] \\
\hline $\begin{array}{l}\text { c.IVS5+1G }>\mathrm{T} \\
\text { (c.936+1G >T) }\end{array}$ & $\mathrm{n} / \mathrm{a}$ & $1^{\circ}-2^{\circ}$ AVB, VT, PM & Coil 2 & {$[4,56,64]$} \\
\hline c. $937-11 C>G$ & p.Leu313GlyfsX31 & $1^{\circ}-3^{\circ} \mathrm{AVB}, \mathrm{LGMD}(1 \mathrm{~B}), \mathrm{VT}, \mathrm{AF}$ & Coil 2 & [65] \\
\hline c. $949 \mathrm{G}>\mathrm{A}$ & p.Glu317Lys & 1\%/3 AVB, AFL, LBBB, HF & Coil 2 & {$[4,5,35,34]$} \\
\hline c. $952 \mathrm{G}>\mathrm{A}$ & p.Ala318Thr & TC, PVB, HF & Coil 2 & [31] \\
\hline c.958delC & p.Leu320fs & $\mathrm{n} / \mathrm{a}$ & Coil 2 & [17] \\
\hline c.959delT & p.Leu320fs X160 & $\begin{array}{l}2^{\circ}-3^{\circ} \text { AVB, LBBB, AF, VT, SVT, } \\
\text { PVB, LGMD, EDMD, HF, SCD }\end{array}$ & Coil 2 & {$[66,67]$} \\
\hline c. $961 \mathrm{C}>\mathrm{T}$ & p.Arg321X & $1^{\circ} \mathrm{AVB}, \mathrm{RBBB}, \mathrm{AF}, \mathrm{VT}, \mathrm{HF}$ & Coil 2 & {$[68,69]$} \\
\hline $\begin{array}{l}\text { c. }[992 \mathrm{G}>\mathrm{A} ;=]+[=; \\
1039 \mathrm{G}>\mathrm{A}]\end{array}$ & 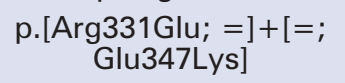 & $A F, P M$ & Coil 2 & [58] \\
\hline c. $992 \mathrm{G}>\mathrm{C}$ & p.Arg331Pro & AVB, PM, LGMD & Coil 2 & [58] \\
\hline c. $992 \mathrm{G}>\mathrm{A}$ & p.Arg331GIn & $1^{\circ} \mathrm{AVB}, \mathrm{AFL}, \mathrm{VT}, \mathrm{HF}$ & Coil 2 & {$[69]$} \\
\hline c. $1003 \mathrm{C}>\mathrm{T}$ & p.Arg335Trp & $3^{\circ} \mathrm{AVB}, \mathrm{RBBB}, \mathrm{SSS}, \mathrm{AF}, \mathrm{VT}$ & Coil 2 & {$[17,70]$} \\
\hline c. $1004 \mathrm{G}>\mathrm{A}$ & p.Arg335GIn & $\mathrm{n} / \mathrm{a}$ & Coil 2 & [37] \\
\hline c. $1039 \mathrm{G}>\mathrm{A}$ & p.Glu347Lys & $A F, P M, S C D$ & Coil 2 & {$[13,71]$} \\
\hline c. $1044 \mathrm{G}>\mathrm{T}$ & p.Met348Ile & EDMD, PM, CCD & Coil 2 & [72] \\
\hline c. $1045 \mathrm{C}>\mathrm{T}$ & p.Arg349Trp & CCD, SVA, MD, HF, SCD & Coil 2 & [8] \\
\hline c. $1046 \mathrm{G}>\mathrm{T}$ & p.Arg349Leu & $\mathrm{AF}, \mathrm{HF}$ & Coil 2 & [73] \\
\hline c. $1048 \mathrm{G}>\mathrm{C}$ & p.Ala350Pro & AVB, LBBB, AF, PVB & Coil 2 & [18] \\
\hline c. $1057 \mathrm{C}>\mathrm{A}$ & p.GIn353Lys & $\mathrm{CA}, \mathrm{MP}, \mathrm{HF}$ & Coil 2 & {$[48]$} \\
\hline c. $1063 \mathrm{C}>\mathrm{T}$ & p.GIn355X & $2^{\circ} \mathrm{AVB}, \mathrm{AF}, \mathrm{VT}, \mathrm{HF}$ & Coil 2 & {$[7,69]$} \\
\hline c. $1069 \mathrm{G}>\mathrm{C}$ & p.Asp357His & $\mathrm{AVB}, \mathrm{VT}$ & Coil 2 & [74] \\
\hline c. $1070 \mathrm{~A}>\mathrm{C}$ & p.Asp357Ala & $\begin{array}{c}2^{\circ} \mathrm{AVB}, \mathrm{Br}, \mathrm{AF}, \mathrm{VES}, \mathrm{VT}, \\
\mathrm{VF}, \mathrm{HF}, \mathrm{SCD}\end{array}$ & Coil 2 & [70] \\
\hline c. $1072 \mathrm{G}>\mathrm{T}$ & p.Glu358X & $1^{\circ}-2^{\circ} \mathrm{AVB}, \mathrm{VT}$ & Coil 2 & [75] \\
\hline c.1085_1085delT & p.Leu363TrpfsX117 & $1^{\circ}-2^{\circ} \mathrm{AVB}, \mathrm{AF}, \mathrm{VT}, \mathrm{HF}$ & Coil 2 & [39] \\
\hline $\begin{array}{l}\text { c. } 1102 \text { 1130dupGCCCTG } \\
\text { GACATGGAGATCCACGC } \\
\text { CTACCG }\end{array}$ & p.Lys378ProfsX112 & LBBB, VT, LGMD, HF & Coil 2 & [76] \\
\hline c.1111_1125del15 & p.Met371_Ala375del & $\mathrm{n} / \mathrm{a}$ & Coil 2 & [17] \\
\hline c.1114̄elelG & p.Glu372ÄrgfsX107 & $1^{\circ} \mathrm{AVB}, \mathrm{Br}, \mathrm{AF}, \mathrm{HF}$ & Coil 2 & [31] \\
\hline c. $1129 \mathrm{C}>\mathrm{T}$ & p.Arg377Cys & LGMD, HF & Coil 2 & [77] \\
\hline c. $1130 \mathrm{G}>\mathrm{A}$ & p.Arg377His & $\begin{array}{l}\text { 10-3o AVB, LBBB, RBBB, } \\
\text { AF, PVB, VT, VF, LGMD, } \\
\text { EDMD, HF, SCD }\end{array}$ & Coil 2 & $\begin{array}{c}{[3,8,18,30,} \\
41,78]\end{array}$ \\
\hline c. $1130 \mathrm{G}>\mathrm{T}$ & p.Arg377Leu & $\begin{array}{c}\left(2^{\circ}\right) \text { AVB, Br, SSS, AF, AFL, } \\
\text { ASS, ATC, VT, LGMD (1B), } \\
\text { EDMD, HF, SCD }\end{array}$ & Coil 2 & {$[4,8,79,80]$} \\
\hline c. $1157 \mathrm{G}>\mathrm{C}$ & p.Arg386Thr & FPLD, CCD & Tail & [81] \\
\hline c. $1157+1 \mathrm{G}>\mathrm{A}$ & $\mathrm{n} / \mathrm{a}$ & VT, VF & Tail & [4] \\
\hline c. $1157+1 \mathrm{G}>\mathrm{T}$ & p.Arg386SerfsX21 & VES, VF, HF & Tail & [70] \\
\hline c. $1163 \mathrm{G}>\mathrm{A}$ & p.Arg $388 \mathrm{His}$ & $\mathrm{Br}, \mathrm{PVB}, \mathrm{HF}, \mathrm{SCD}$ & Tail & [31] \\
\hline c. $1189 \mathrm{C}>\mathrm{T}$ & p.Arg397Cys & $\mathrm{n} / \mathrm{a}$ & Tail & [37] \\
\hline c. $1195 \mathrm{C}>\mathrm{T}$ & p.Arg399Cys & HF & Tail & [31] \\
\hline c.1197_1240del44 & p.Gly400Argfs*11 & AVB, SSS & Tail & [55] \\
\hline c. $1292 \bar{C}>\mathrm{G}$ & p.Ser $431^{*}$ & AVB & Tail & [55] \\
\hline c. $1294 C>T$ & p.GIn432X & $3^{\circ} \mathrm{AVB}, \mathrm{LBBB}, \mathrm{RBBB}, \mathrm{AF}, \mathrm{VT}, \mathrm{HF}$ & Tail & [69] \\
\hline c. $1303 \mathrm{C}>\mathrm{T}$ & p.Arg435Cys & $\mathrm{HF}$ & Tail & [13] \\
\hline c.1307_1308insGCAC & p.Ser437HisfsX1 & $\begin{array}{l}2^{\circ} \mathrm{AVB}, \mathrm{Br}, \mathrm{TC}, \mathrm{AF}, \\
\mathrm{PVB}, \mathrm{HF}, \mathrm{SCD}\end{array}$ & Tail & [31] \\
\hline
\end{tabular}




\begin{tabular}{|c|c|c|c|c|}
\hline Nucleotide change & Protein change & DCM phenotype range & Domain & Reference* \\
\hline c. $1318 \mathrm{G}>\mathrm{A}$ & p.Val440Met & LAFB, VES & Tail & [69] \\
\hline c.1370delA & p.Lys457SerfsX21 & AF, LGMD & Tail & [58] \\
\hline c. $1380+1 G>A$ & $\mathrm{n} / \mathrm{a}$ & $\left(1^{\circ}\right) \mathrm{AVB}, \mathrm{AF}, \mathrm{VF}, \mathrm{HF}$ & Tail & {$[8,34]$} \\
\hline c.1397_1397delA & p.Asn466IlefsX14 & $\begin{array}{l}1^{\circ} \mathrm{AVB}, \mathrm{LBBB}, \mathrm{Br}, \mathrm{AF}, \\
\text { VF, VT, HF, SCD }\end{array}$ & Tail & {$[41,82]$} \\
\hline c. $1412 \mathrm{G}>\mathrm{A}$ & p.Arg 471His & $\mathrm{AF}, \mathrm{VT}, \mathrm{HF}$ & Tail & [31] \\
\hline c.1424_1425insAGA & p.Gly474_Asp475insGlu & HF, ICD & Tail & [31] \\
\hline c. $1443 C>G$ & p.Tyr481X & $2^{\circ} \mathrm{AVB}, \mathrm{RBBB}, \mathrm{SVT}, \mathrm{VT}$, HF & Tail & {$[46,48,49]$} \\
\hline c. $1489-1 \mathrm{G}>\mathrm{T}$ & p. Ile497-Glu536del & $\begin{array}{c}2^{\circ} \mathrm{AVB}, \mathrm{Br}, \mathrm{AF}, \mathrm{AFL} \\
\text { VT, } \mathrm{EDMD}\end{array}$ & Tail & {$[70]$} \\
\hline c. $1492 \mathrm{~T}>\mathrm{A}$ & p.Trp498Arg & AVB, EDMD(2) & Tail & [4] \\
\hline c.1493_1493delG & p.Ala499LeufsX47 & $\mathrm{AF}, \mathrm{VT}, \mathrm{HF}$ & Tail & [39] \\
\hline c.1496delC & p.Ala499Val & $\begin{array}{c}1^{\circ}-2^{\circ} \text { AVB, RBBB, Br, } \\
\text { ATC, ASS, AN }\end{array}$ & Tail & [83] \\
\hline c.1512_1513insAG & p.Thr505ArgfsX44 & $1^{\circ} \mathrm{AVB}, \mathrm{AF}, \mathrm{PVB}, \mathrm{HF}, \mathrm{SCD}$ & Tail & [8] \\
\hline c.1526_1527insC & p.Thr510TyrfsX42 & $1^{\circ} \mathrm{AVB} / \mathrm{AVB}, \operatorname{EDMD}(2)$ & Tail & [4] / [84] \\
\hline c. $1549 \mathrm{C}>\mathrm{T}$ & p.GIn517X & $\begin{array}{c}3^{\circ} \text { AVB, } A F, \text { EDMD, } \\
\text { VF, SCD }\end{array}$ & Tail & [70] \\
\hline c. $1560 \mathrm{G}>\mathrm{A}$ & p.Trp520X & $2^{\circ} \mathrm{AVB}, \mathrm{LBBB}, \mathrm{Br}$ & Tail & {$[70]$} \\
\hline c. $1567 \mathrm{G}>\mathrm{A}$ & p.Gly523Arg & LBBB & Tail & {$[33,34]$} \\
\hline c.1579_1580insCTGC & p.Arg527ProfsX26 & $1^{\circ}-2^{\circ} \mathrm{AVB}, \mathrm{LBBB}, \mathrm{LAFB}, \mathrm{HF}$ & Tail & {$[4,5,35]$} \\
\hline c. $1583 C>T$ & p.Thr528Met & $1^{\circ}-3^{\circ} \mathrm{AVB}, \mathrm{Br}, \mathrm{AF}, \mathrm{SVA}$ & Tail & [10] \\
\hline c. $1608+1 \mathrm{G}>\mathrm{T}$ & $\mathrm{n} / \mathrm{a}$ & $\mathrm{n} / \mathrm{a}$ & Tail & [37] \\
\hline $\begin{array}{l}\text { c.IVS9-3C>G } \\
\text { (c. } 1609-3 C>G \text { ) }\end{array}$ & Loss of exon 10 & $3^{\circ} \mathrm{AVB}, \mathrm{Br}, \mathrm{PM}, \mathrm{LGMD}(1 \mathrm{~B})$ & Tail & [85] \\
\hline c. $1621 \mathrm{C}>\mathrm{T}$ & p.Arg541Cys & $\begin{array}{l}\text { LBBB, VT, VF, PVB, } \\
\text { fibrosis, SWMA, SCD }\end{array}$ & Tail & [86-88] \\
\hline c. $1621 \mathrm{C}>\mathrm{A}$ & p.Arg541Ser & VT, HF / LGMD(1B), HF & Tail & $\begin{array}{l}{[16] /[46,} \\
39,48]\end{array}$ \\
\hline c. $1621 C>G$ & p.Arg541Gly & $\begin{array}{l}\text { IVB, LBBB, Br, SVA, } \\
\text { PVB, VA, TC }\end{array}$ & Tail & [89] \\
\hline c. $1622 \mathrm{G}>\mathrm{A}$ & p.Arg541His & AVB, AF, PVB & Tail & [18] \\
\hline c. $1622 \mathrm{G}>\mathrm{C}$ & p.Arg541Pro & PVB, VT, HF & Tail & {$[8]$} \\
\hline c. $1711 \mathrm{C}>\mathrm{A}$ & p.Arg571Ser & $1^{\circ}-3^{\circ} \mathrm{AVB}, \mathrm{Br}, \mathrm{AF}$ & Tail & [23] \\
\hline c. $1713 \mathrm{C}>\mathrm{A}$ & p.Ser571Arg & $2^{\circ}, 3^{\circ} \mathrm{AVB}, \mathrm{PM}, \mathrm{HF}, \mathrm{AF}$ & Tail & [23] \\
\hline c.1714insCTGC & p.Ser572LeufsX8 & $1^{\circ}, 2^{\circ} \mathrm{AVB}, \mathrm{PM}, \mathrm{LBBB}$ & Tail & [35] \\
\hline c. $1718 \mathrm{C}>\mathrm{T}$ & p.Ser573Leu & AVB, VT & Tail & {$[4,30]$} \\
\hline c. $1904 \mathrm{G}>\mathrm{A}$ & p.Gly645Asp & $\mathrm{HF}$ & Tail & [90] \\
\hline c. $1930 \mathrm{C}>\mathrm{T}$ & p.Arg644Cys & $\begin{array}{c}\text { AVB, AF, VT, HF / LGMD(1B), } \\
\text { HF, SCD }\end{array}$ & Tail & $\begin{array}{c}{[4,43} \\
69] /[91]\end{array}$ \\
\hline c. $1960 \mathrm{C}>\mathrm{T}$ & p.Arg654X & $\begin{array}{c}\text { AVB, LBBB, SSS, AF, } \\
\text { VT, HF, SCD }\end{array}$ & Tail & [31] \\
\hline c.1964_1965insG & p.Thr655fsX49 & PM, VT, MH, POS, HF, SCD & Tail & [92] \\
\hline $\begin{array}{l}\text { c. }[1699 \text { to } 183 \text { 1699- } \\
\text { 160inv24; } 568-1699- \\
\text { 184del; } 1699 \text { to } \\
\text { 159_1995+6997del] }\end{array}$ & $\begin{array}{l}\text { Double deletion with break } \\
\text { points in exon } 3 \text {, intron } 10, \\
\text { downstream of gene }\end{array}$ & $\begin{array}{c}1^{\circ}-3^{\circ} \mathrm{AVB}, \mathrm{AF}, \mathrm{AFL} \\
\mathrm{Br}, \mathrm{VA}, \mathrm{SCD}\end{array}$ & Coil/Tail & [93] \\
\hline Deletion exons 3-12 & Deletion $>4,704 \mathrm{bp}$ & VT, HF & Coil/Tail & [48] \\
\hline
\end{tabular}

$1^{\circ}, 2^{\circ}, 3^{\circ}$ - atrio-ventricular block degree, in parenthesis when degree specified only in some studies; AF — atrial fibrillation; AFL — atrial flutter; AN - axonal neuropathy; ASS - atrial standstill; ATC — atrial tachycardia; AVB — atrio-ventricular block; Br - bradycardia;

CA - cardiac abnormalities; CCD — cardiac conduction disease; CMT2 - Charcot-Marie-Tooth disease; DCM - dilated cardiomyopathy;

$\mathrm{DM}$ - diabetes mellitus; EDMD(2) - Emery-Dreifuss muscular dystrophy (type 2); FPLD — familial partial lipodystrophy; HF - heart failure; IVB - intra-ventricular block; ICD — implantable cardiac defibrillator; LAFB - left anterior fascicular block; LBBB — left bundle branch block LGMD(1B) - limb girdle muscular dystrophy (type 1B); LVE — left ventricular exosystoly; MA — muscular atrophy; MAD — mandibuloacral dysplasia; MD - muscular dystrophy; $\mathrm{MH}$ - muscular hypertrophy; MP — myopathy; n/a — not available; PAB - premature atrial beats; PM - pacemaker implantation; PN - peripheral neuropathy; POS - polycystic ovary syndrome; PVB - premature ventricular beats; RBBB - right bundle branch block; SCD — sudden cardiac death; SSS - sick sinus syndrome; SVA — supraventricular arrhythmia; SVT - supraventricular tachycardia; SWMA — segmental wall motion abnormalities; TC — tachycardia; VA — ventricular arrhythmia; VES - ventricular extra systoles; VF — ventricular fibrillation; VT — ventricular tachycardia; LD - lipodystrophy; WS — Werner syndrome; "I" used to separate differing phenotypes; *References are listed in "Supplement 1" 


\section{SUPPLEMENT 1: Table 1 References}

1. van Tintelen JP, Tio RA, Kerstjens-Frederikse WS et al. Severe myocardial fibrosis caused by a deletion of the 5' end of the lamin A/C gene. J Am Coll Cardiol, 2007; 49: 2430-2439.

2. Becane HM, Bonne G, Varnous S et al. High incidence of sudden death with conduction system and myocardial disease due to lamins A and C gene mutation. Pacing Clin Electrophysiol, 2000; 23: 1661-1666.

3. Sebillon P, Bouchier C, Bidot LD et al. Expanding the phenotype of LMNA mutations in dilated cardiomyopathy and functional consequences of these mutations. J Med Genet, 2003; 40: 560-567.

4. Pasotti M, Klersy C, Pilotto A et al. Long-term outcome and risk stratification in dilated cardiolaminopathies. J Am Coll Cardiol, 2008; 52: 1250-1260.

5. Verga L, Concardi M, Pilotto A et al. Loss of lamin A/C expression revealed by immuno-electron microscopy in dilated cardiomyopathy with atrioventricular block caused by LMNA gene defects. Virchows Arch, 2003; 443: 664-671.

6. Ho JC, Zhou T, Lai WH et al. Generation of induced pluripotent stem cell lines from 3 distinct laminopathies bearing heterogeneous mutations in lamin A/C. Aging (Albany NY), 2011; 3: 380-390.

7. Pethig K, Genschel J, Peters T et al. LMNA mutations in cardiac transplant recipients. Cardiology, 2005; 103: 57-62.

8. van Tintelen JP, Hofstra RM, Katerberg $\mathrm{H}$ et al. High yield of LMNA mutations in patients with dilated cardiomyopathy and/or conduction disease referred to cardiogenetics outpatient clinics. Am Heart J, 2007; 154: 1130-1139.

9. Yuan WL, Huang CY, Wang JF et al. R25G mutation in exon 1 of LMNA gene is associated with dilated cardiomyopathy and limb-girdle muscular dystrophy 1B. Chin Med J (Engl), 2009; 122: 2840-2845.

10. Saj M, Dabrowski R, Labib S et al. Variants of the lamin A/C (LMNA) gene in non-valvular atrial fibrillation patients: A possible pathogenic role of the Thr528Met mutation. Mol Diagn Ther, 2012; 16: 99-107.

11. Garg A, Speckman RA, Bowcock AM. Multisystem dystrophy syndrome due to novel missense mutations in the amino-terminal head and alpha-helical rod domains of the lamin $\mathrm{A} / \mathrm{C}$ gene. Am J Med, 2002; 112: 549-555.

12. Vytopil M, Ricci E, Dello RA et al. Frequent low penetrance mutations in the Lamin A/C gene, causing Emery Dreifuss muscular dystrophy. Neuromuscul Disord, 2002; 12: 958-963.

13. Vytopil M, Benedetti S, Ricci E et al. Mutation analysis of the lamin $\mathrm{A} / \mathrm{C}$ gene (LMNA) among patients with different cardiomuscular phenotypes. J Med Genet, 2003; 40: e132.

14. Goizet C, Yaou RB, Demay Let al. A new mutation of the lamin $\mathrm{A} / \mathrm{C}$ gene leading to autosomal dominant axonal neuropathy, muscular dystrophy, cardiac disease, and leuconychia. J Med Genet, 2004; 41: e29.

15. Arbustini E, Pilotto A, Grasso M et al. Novel human pathological mutations. Gene symbol: LMNA. Disease: cardiomyopathy, dilated with conduction defects. Hum Genet, 2009; 125: 350.

16. Scharner J, Brown CA, Bower M et al. Novel LMNA mutations in patients with Emery-Dreifuss muscular dystrophy and functional characterization of four LMNA mutations. Hum Mutat, 2011; 32: 152-167.

17. Lakdawala NK, Funke BH, Baxter S et al. Genetic testing for dilated cardiomyopathy in clinical practice. J Card Fail, 2012; 18: 296-303.
18. Rudenskaya GE, Polyakov AV, Tverskaya SM et al. Laminopathies in Russian families. Clin Genet, 2008; 74: 127-133.

19. Song K, Dube MP, Lim J, Hwang I, Lee I, Kim JJ. Lamin A/C mutations associated with familial and sporadic cases of dilated cardiomyopathy in Koreans. Exp Mol Med, 2007; 39: 114-120.

20. Chen L, Lee L, Kudlow BA et al. LMNA mutations in atypical Werner's syndrome. Lancet, 2003; 362: 440-445.

21. Nguyen D, Leistritz DF, Turner L et al. Collagen expression in fibroblasts with a novel LMNA mutation. Biochem Biophys Res Commun, 2007; 352: 603-608.

22. McPherson E, Turner L, Zador I, Reynolds K, MacGregor D, Giampietro PF. Ovarian failure and dilated cardiomyopathy due to a novel lamin mutation. Am J Med Genet A, 2009; 149A: 567-572.

23. Fatkin D, MacRae C, Sasaki T et al. Missense mutations in the rod domain of the lamin $\mathrm{A} / \mathrm{C}$ gene as causes of dilated cardiomyopathy and conduction-system disease. N Engl J Med, 1999; 341: 1715-1724.

24. van der Kooi AJ, Bonne G et al. Lamin A/C mutations with lipodystrophy, cardiac abnormalities, and muscular dystrophy. Neurology, 2002; 59: 620-623.

25. Carboni N, Porcu M, Mura M et al. Evolution of the phenotype in a family with an LMNA gene mutation presenting with isolated cardiac involvement. Muscle Nerve, 2010; 41: 85-91.

26. Subramanyam L, Simha V, Garg A. Overlapping syndrome with familial partial lipodystrophy, Dunnigan variety and cardiomyopathy due to amino-terminal heterozygous missense lamin $\mathrm{A} / \mathrm{C}$ mutations. Clin Genet, 2010; 78: 66-73.

27. Arbustini Eloisa AE, Pilotto A et al. Gene symbol: LMNA. Disease: EDMD2. Hum Genet, 2005; 117: 294.

28. Wang $\mathrm{H}$, Wang J, Zheng $\mathrm{W}$ et al. Mutation Glu82Lys in lamin $\mathrm{A} / \mathrm{C}$ gene is associated with cardiomyopathy and conduction defect. Biochem Biophys Res Commun, 2006; 344: 17-24.

29. Wu X, Wang QK, Gui $\mathrm{L}$ et al. Identification of a new lamin $\mathrm{A} / \mathrm{C}$ mutation in a chinese family affected with atrioventricular block as the prominent phenotype. J Huazhong Univ Sci Technolog Med Sci, 2010; 30: 103-107.

30. Taylor MR, Fain PR, Sinagra G et al. Natural history of dilated cardiomyopathy due to lamin A/C gene mutations. J Am Coll Cardiol, 2003; 41: 771-780.

31. Parks SB, Kushner JD, Nauman D et al. Lamin A/C mutation analysis in a cohort of 324 unrelated patients with idiopathic or familial dilated cardiomyopathy. Am Heart J, 2008; 156: 161-169.

32. Redondo-Verge L, Yaou RB, Fernandez-Recio M, Dinca L, Richard P, Bonne G. Cardioembolic stroke prompting diagnosis of LMNA-associated Emery-Dreifuss muscular dystrophy. Muscle Nerve, 2011; 44: 587-589.

33. Millat G, Chanavat V, Julia S, Crehalet H, Bouvagnet P, Rousson R. Validation of high-resolution DNA melting analysis for mutation scanning of the LMNA gene. Clin Biochem, 2009; 42: 892-898.

34. Millat G, Bouvagnet $\mathrm{P}$, Chevalier $\mathrm{P}$ et al. Clinical and mutational spectrum in a cohort of 105 unrelated patients with dilated cardiomyopathy. Eur J Med Genet, 2011; 54: e570-e575.

35. Arbustini E, Pilotto A, Repetto A et al. Autosomal dominant dilated cardiomyopathy with atrioventricular block: A lamin A/C defect-related disease. J Am Coll Cardiol, 2002; 39: 981-990.

36. Pan H, Richards AA, Zhu X, Joglar JA, Yin HL, Garg V. A novel mutation in LAMIN A/C is associated with isolated early-onset atrial fibrillation and progressive atrioventricular block followed by cardiomyopathy and sudden cardiac death. Heart Rhythm, 2009; 6: 707-710. 
37. Narula N, Favalli V, Tarantino P et al. Quantitative expression of the mutated lamin $\mathrm{A} / \mathrm{C}$ gene in patients with cardiolaminopathy. J Am Coll Cardiol, 2012; 60: 1916-1920.

38. Keller H, Finsterer J, Steger C et al. Novel c.367_369del LMNA mutation manifesting as severe arrhythmias, dilated cardiomyopathy, and myopathy. Heart Lung, 2012; 41: 382-386.

39. Karkkainen S, Reissell E, Helio T et al. Novel mutations in the lamin $\mathrm{A} / \mathrm{C}$ gene in heart transplant recipients with end stage dilated cardiomyopathy. Heart, 2006; 92: 524-526.

40. Lupsa BC, Sachdev V, Lungu AO, Rosing DR, Gorden P. Cardiomyopathy in congenital and acquired generalized lipodystrophy: A clinical assessment. Medicine (Baltimore), 2010; 89: 245-50.

41. Perrot A, Sigusch HH, Nagele $\mathrm{H}$ et al. Genetic and phenotypic analysis of dilated cardiomyopathy with conduction system disease: Demand for strategies in the management of presymptomatic lamin A/C mutant carriers. Eur J Heart Fail, 2006; 8: 484-493.

42. Karkkainen S, Helio T, Miettinen R et al. A novel mutation, Ser143 Pro, in the lamin $\mathrm{A} / \mathrm{C}$ gene is common in finnish patients with familial dilated cardiomyopathy. Eur Heart J, 2004; 25: 885-893.

43. Perrot $\mathrm{A}$, Hussein $\mathrm{S}$, Ruppert $\mathrm{V}$ et al. Identification of mutational hot spots in LMNA encoding lamin $\mathrm{A} / \mathrm{C}$ in patients with familial dilated cardiomyopathy. Basic Res Cardiol, 2009; 104: 90-99.

44. Saga A, Karibe A, Otomo J et al. Lamin A/C gene mutations in familial cardiomyopathy with advanced atrioventricular block and arrhythmia. Tohoku J Exp Med, 2009; 218: 309-316.

45. Botto N, Vittorini S, Colombo MG et al. A novel LMNA mutation (R189W) in familial dilated cardiomyopathy: Evidence for a ,hot spot' region at exon 3: A case report. Cardiovasc Ultrasound, 2010; 8: 9 .

46. Sylvius N, Bilinska ZT, Veinot JP et al. In vivo and in vitro examination of the functional significances of novel lamin gene mutations in heart failure patients. J Med Genet, 2005; 42: 639-647.

47. Fernandez X, Dumont CA, Monserrat L, Hermida-Prieto M, Castro-Beiras A. Sudden death in a patient with lamin A/C gene mutation and near normal left ventricular systolic function. Int J Cardiol, 2008; 126: 136-137.

48. Gupta P, Bilinska ZT, Sylvius N et al. Genetic and ultrastructural studies in dilated cardiomyopathy patients: A large deletion in the lamin $\mathrm{A} / \mathrm{C}$ gene is associated with cardiomyocyte nuclear envelope disruption. Basic Res Cardiol, 2010; 105: 365-377.

49. Bilinska ZT, Sylvius N, Grzybowski J et al. Dilated cardiomyopathy caused by LMNA mutations. Clinical and morphological studies. Kardiol Pol, 2006; 64: 812-819.

50. Jakobs PM, Hanson EL, Crispell KA et al. Novel lamin A/C mutations in two families with dilated cardiomyopathy and conduction system disease. J Card Fail, 2001; 7: 249-256.

51. Cowan J, Li D, Gonzalez-Quintana J, Morales A, Hershberger RE. Morphological analysis of 13 LMNA variants identified in a cohort of 324 unrelated patients with idiopathic or familial dilated cardiomyopathy. Circ Cardiovasc Genet, 2010; 3: 6-14.

52. Otomo J, Kure S, Shiba T et al. Electrophysiological and histopathological characteristics of progressive atrioventricular block accompanied by familial dilated cardiomyopathy caused by a novel mutation of lamin $\mathrm{A} / \mathrm{C}$ gene. J Cardiovasc Electrophysiol, 2005; 16: 137-145.

53. Hershberger RE, Hanson EL, Jakobs PM et al. A novel lamin $\mathrm{A} / \mathrm{C}$ mutation in a family with dilated cardiomyopathy, prominent conduction system disease, and need for permanent pacemaker implantation. Am Heart J, 2002; 144: 1081-1086.
54. Arbustini E, Pasotti M, Pilotto A et al. Gene symbol: LMNA. Disease: Dilated cardiomyopathy with conduction defect CMD1A. Hum Genet, 2007; 120:910.

55. Saj M, Bilinska ZT, Tarnowska A et al. LMNA mutations in Polish patients with dilated cardiomyopathy: Prevalence, clinical characteristics, and in vitro studies. BMC Med Genet, 2013; 23:55.

56. Arbustini Eloisa AE, Pilotto A, Pasotti M et al. Gene symbol: LMNA. Disease: Cardiomyopathy, dilated, with conduction defect 1. Hum Genet, 2005; 117: 298.

57. Carboni N, Mura M, Marrosu G et al. Muscle MRI findings in patients with an apparently exclusive cardiac phenotype due to a novel LMNA gene mutation. Neuromuscul Discord, 2008; 18: 291-298.

58. Benedetti S, Menditto I, Degano M et al. Phenotypic clustering of lamin A/C mutations in neuromuscular patients. Neurology, 2007; 69: 1285-1292.

59. Kichuk Chrisant MR, Drummond-Webb J, Hallowell S, Friedman NR. Cardiac transplantation in twins with autosomal dominant Emery-Dreifuss muscular dystrophy. J Heart Lung Transplant, 2004; 23: 496-498.

60. Beckmann BM, Holinski-Feder E, Walter MC et al. Laminopathy presenting as familial atrial fibrillation. Int J Cardiol, 2010; 145: 394-396.

61. MacLeod HM, Culley MR, Huber JM, McNally EM. Lamin A/C truncation in dilated cardiomyopathy with conduction disease. BMC Med Genet, 2003; 4:4.

62. Antoniades L, Eftychiou C, Kyriakides T, Christodoulou K, Katritsis DG. Malignant mutation in the lamin A/C gene causing progressive conduction system disease and early sudden death in a family with mild form of limb-girdle muscular dystrophy. J Interv Card Electrophysiol, 2007; 19:1-7.

63. Ben Yaou R, Bécane HM, Demay L et al. La dystrophie musculaire des ceintures autosomique dominante associée à des troubles de la conduction cardiaque (LGMD1B). Description de 8 nouvelles familles avec mutations du gène LMNA. Rev Neurol (Paris), 2005; 161: 42-54.

64. Arbustini E, Pilotto A, Porcu E et al. Gene symbol: LMNA. Disease: Cardiomyopathy, dilated, with conduction tissue defect 1. Hum Genet, 2005; 117: 300.

65. Carboni N, Floris M, Mateddu A et al. Aberrant splicing in the LMNA gene caused by a novel mutation on the polypyrimidine tract of intron 5. Muscle Nerve, 2011; 43: 688-693.

66. Brodsky GL, Muntoni F, Miocic S, Sinagra G, Sewry C, Mestroni L. Lamin A/C gene mutation associated with dilated cardiomyopathy with variable skeletal muscle involvement. Circulation, 2000; 101: 473-476.

67. Raffaele DB, Ricci E, Galluzzi G et al. Different mutations in the LMNA gene cause autosomal dominant and autosomal recessive Emery-Dreifuss muscular dystrophy. Am J Hum Genet, 2000; 66: 1407-1412.

68. Zeller R, Ivandic BT, Ehlermann P et al. Large-scale mutation screening in patients with dilated or hypertrophic cardiomyopathy: A pilot study using DGGE. J Mol Med (Berl), 2006; 84: 682-691.

69. Moller DV, Pham TT, Gustafsson F et al. The role of Lamin A/C mutations in Danish patients with idiopathic dilated cardiomyopathy. Eur J Heart Fail, 2009; 11: 1031-1035.

70. Stallmeyer B, Koopmann M, Schulze-Bahr E. Identification of novel mutations in LMNA associated with familial forms of dilated cardiomyopathy. Genet Test Mol Biomarkers, 2012; 16: 543-549. 
71. Benedetti S, Menditto I, Degano M et al. Phenotypic clustering of lamin $\mathrm{A} / \mathrm{C}$ mutations in neuromuscular patients. Neurology, 2007; 69: 1285-1292.

72. Meinke P, Nguyen TD, Wehnert MS. The LINC complex and human disease. Biochem Soc Trans, 2011; 39: 1693-1697.

73. Hermida-Prieto M, Monserrat L, Castro-Beiras A et al. Familial dilated cardiomyopathy and isolated left ventricular noncompaction associated with lamin $\mathrm{A} / \mathrm{C}$ gene mutations. Am J Cardiol, 2004; 94: 50-54.

74. Fujimori Y, Okimatsu H, Kashiwagi T et al. Molecular defects associated with antithrombin deficiency and dilated cardiomyopathy in a Japanese patient. Intern Med, 2008; 47: 925-931.

75. De Backer J, Van Beeumen K, Loeys B, Duytschaever M. Expanding the phenotype of sudden cardiac death: An unusual presentation of a family with a Lamin A/C mutation. Int J Cardiol, 2010; 138: 97-99.

76. Volpi L, Ricci G, Passino C et al. Prevalent cardiac phenotype resulting in heart transplantation in a novel LMNA gene duplication. Neuromuscul Disord, 2010; 20: 512-516.

77. Komaki H, Hayashi YK, Tsuburaya R et al. Inflammatory changes in infantile-onset LMNA-associated myopathy. Neuromuscul Disord, 2011; 21: 563-568.

78. Charniot JC, Pascal C, Bouchier $\mathrm{C}$ et al. Functional consequences of an LMNA mutation associated with a new cardiac and non-cardiac phenotype. Hum Mutat, 2003; 21: 473-481.

79. Boriani G, Gallina M, Merlini L et al. Clinical relevance of atrial fibrillation/flutter, stroke, pacemaker implant, and heart failure in Emery-Dreifuss muscular dystrophy: A long-term longitudinal study. Stroke, 2003; 34: 901-908.

80. HongJS, Ki CS, KimJW etal. Cardiac dysrhythmias,cardiomyopathy and muscular dystrophy in patients with Emery-Dreifuss muscular dystrophy and limb-girdle muscular dystrophy type 1B. J Korean Med Sci, 2005; 20: 283-290.

81. Balavoine AS, Laurent JM, Brigadeau F, Lascols O, Vantyghem MC. Atteinte cardiaque et diabète lié à une mutation de la lamine $\mathrm{A} / \mathrm{C}$ habituellement rencontrée dans les dystrophies musculaires d'Emery Dreifuss. Congress Abstract P206. 36ième congrès annuel de la société francophone du diabète. Diab Metab, 2010; 36: A87.

82. Genschel J, Baier P, Kuepferling S et al. A new frameshift mutation at codon 466 (1397delA) within the LMNA gene. Hum Mutat, 2000; 16: 278 .

83. Duparc A, Cintas P, Somody E et al. A cardio-neurological form of laminopathy: dilated cardiomyopathy with permanent partial atrial standstill and axonal neuropathy. Pacing Clin Electrophysiol, 2009; 32: 410-415.

84. Arbustini EA, Pasotti M, Pilotto A, Repetto A, Grasso M, Diegoli M. Gene symbol: CMD1A. Disease: Dilated cardiomyopathy as- sociated with conduction system disease. Hum Genet, 2005; 117: 295.

85. Chrestian N, Valdmanis PN, Echahidi N et al. A novel mutation in a large French-Canadian family with LGMD1B. Can J Neurol Sci, 2008; 35: 331-334.

86. Forissier JF, Bonne G, Bouchier C et al. Apical left ventricular aneurysm without atrio-ventricular block due to a lamin $\mathrm{A} / \mathrm{C}$ gene mutation. Eur J Heart Fail, 2003; 5: 821-825.

87. Hookana E, Junttila MJ, Sarkioja T et al. Cardiac arrest and left ventricular fibrosis in a Finnish family with the lamin A/C mutation. J Cardiovasc Electrophysiol, 2008; 19: 743-747.

88. Saj M, Jankowska A, Lewandowski M et al. Dilated cardiomyopathy with profound segmental wall motion abnormalities and ventricular arrhythmia caused by the R541C mutation in the LMNA gene. Int J Cardiol, 2010; 144: e51-e53.

89. Malek LA, Labib S, Mazurkiewicz L et al. A new c.1621 C > G, p.R541G lamin A/C mutation in a family with DCM and regional wall motion abnormalities (akinesis/dyskinesis): genotype-phenotype correlation. J Hum Genet, 2011; 56: 83-86.

90. Duboscq-Bidot L, Charron P, Ruppert V et al. Mutations in the ANKRD1 gene encoding CARP are responsible for human dilated cardiomyopathy. Eur Heart J, 2009; 30: 2128-2136.

91. Mercuri E, Brown SC, Nihoyannopoulos P et al. Extreme variability of skeletal and cardiac muscle involvement in patients with mutations in exon 11 of the lamin A/C gene. Muscle Nerve, 2005; 31: 602-609.

92. Decaudain A, Vantyghem MC, Guerci B et al. New metabolic phenotypes in laminopathies: LMNA mutations in patients with severe metabolic syndrome. J Clin Endocrinol Metab, 2007; 92: 4835-4844.

93. Marsman RF, Bardai A, Postma AV et al. A complex double deletion in LMNA underlies progressive cardiac conduction disease, atrial arrhythmias, and sudden death. Circ Cardiovasc Genet, 2011; 4: 280-287.

94. Chang SH, Tsai CT, Lai LP, Lei MH. Identification of a lamin A/C gene mutation in a Taiwanese family with limb girdle muscular dystrophy and cardiomyopathy. Int J Cardiol, 2010; 145: 598-599.

95. Sparks EA, Boudoulas KD, Raman SV et al. Heritable cardiac conduction and myocardial disease: from the clinic to the basic science laboratory and back to the clinic. Cardiology, 2011; 118: 179-186.

96. Kourgiannidis G, Anastasakis A, Lampropoulos K, Iliopoulos T. A patient with ventricular tachycardia due to a novel mutation of the lamin $\mathrm{A} / \mathrm{C}$ gene: Case presentation and mini review. Hellenic J Cardiol, 2013; 54: 326-330.

97. Muntoni F, Bonne G, Goldfarb LG et al. Disease severity in dominant Emery Dreifuss is increased by mutations in both emerin and desmin proteins. Brain, 2006; 129: 1260-1268. 